\title{
Cytological and Bacteriological Observation of Tracheal Aspirates and Bronchoalveolar Lavage Fluid Obtained from Thoroughbred Racehorses with Pneumonia Associated with Transport
}

\author{
Hirotaka ODE, Seiji HOBO, Yoshinari KATAYAMA, Hidekazu NIWA, Yasushi KUWAMOTO, \\ Tetsuya YAMANE and Toru ANZAI* \\ Epizootic Research Center, Equine Research Institute, Japan Racing Association, 1400-4 Shiba, Shimotsuke- \\ shi, Tochigi 329-0412, Japan
}

TA and BALF obtained from 44 Thoroughbred racehorses that developed pneumonia after long-distance transportation were examined cytologically and bacteriologically. The percentages of neutrophils in both TA and BALF collected from the same horses at the same time were almost the same and were very high indicating inflammation. Various bacteria, including obligative anaerobius which are categorized as opportunistic pathogens, were isolated from $B A L F$, and the bacteria isolated mainly in the later stages of the clinical course tended to be resistant to cephalotine and gentamicin, which are used as the first choice in the racehorse hospital of JRA where this study was performed.

Key words: $B A L F$, horse, pneumonia, $T A$
J. Equine Sci.

Vol. 18, No. 4

pp. 161-165, 2007
Tracheal washing (TW) is a diagnostic procedure used in equine practice for respiratory disease, since tracheal aspirates (TA) can provide clinicians with important cytological and microbiological information $[2,3,11,14,15]$. Bronchoalveolar lavage (BAL) is another diagnostic procedure for respiratory disease in horses, and cytological, biochemical and microbiological examination of BAL fluid (BALF) offers even more accurate information to assist diagnosis of pneumonia [5-7, 9].

Malikides et al. [8] compared TA and BALF cytologically in racehorses that showed poor performance, and concluded that both samples give important information on different regions of the airway [10]. Wood et al. [16] studied the association with inflammatory lower airway disease (IAD) and bacterial infections in British racehorses using TW and an endoscope, and gained evidence that Streptococcus equi subsp. zooepidemicus and Streptococcus pneumoniae play an important etiological role in the pathogenesis of IAD in young horses. Takizawa et al. [15] also

This article was accepted July 12, 2007

*Corresponding author. e-mail: anzai@epizoo.equinst.go.jp performed cytological and bacteriological studies of TA taken from 2-year-old Thoroughbreds transported by vehicle over long distances. They reported that there was no clear difference in the species and number of bacteria between cytologically abnormal and normal samples. The study by Takizawa suggests that bacteriological examination of TA does not always provide information on the etiology of lower respiratory disease in the case of fever associated with transport.

According to these previous studies, BALF may offer more precise information than TA for diagnosis of horses with pneumonia. However, BAL can be done only in equine hospitals, since the bronchoscope that is required to carry out BAL is very expensive and difficult to use outside of the hospital. TA may be easily carried out at the stable. In this study, we compared cytological studies of TA and BALF and analyzed the diagnostic value of bacteria in BALF obtained from Thoroughbred racehorses with pneumonia associated with transport.

All Thoroughbred racehorses examined in this study had developed pneumonia after long-distance transportation, and were showing pyrexia, anorexia, 
Table 1. Cytological findings in the bronchoalveolar lavage fluid and tracheal aspirates obtained from racehorses with pneumonia

\begin{tabular}{llcccc}
\hline Speciment & Rate $(\%)$ & Neutrophils & Macrophages & Lymphocytes & Epithelial cells \\
\hline \multirow{2}{*}{ BALF } & Mean & 73.1 & 14.9 & 9.7 & 2.3 \\
\multirow{2}{*}{ TA } & Range & $30.9-91.4$ & $1.0-45.8$ & $1.8-24.4$ & $0-10.4$ \\
& Mean & 70.6 & 11.5 & 8.9 & 8.9 \\
& Range & $44.6-90.0$ & $3.9-24.6$ & $1.3-33.8$ & $0.9-33.3$ \\
\hline
\end{tabular}

Bronchoalveolar lavage fluid (BALF) was collected from 44 horses, and tracheal aspirates (TA) were collected from 26 of them.

listlessness and abnormal alveolar sound. The horses were young, aged 2 to 3 years old.

The procedure of BAL was performed according to a previous report [9]. Each horse was secured in stocks and sedated with medetomidine hydrochloride (7.5 $\mu \mathrm{g} / \mathrm{kg}$ of body weight, IV). After placement of a nose twitch for restraint and wiping of the horse's nares with gauze, a 270-cm long, 7.9-mm diameter bronchoscope with a $2.0-\mathrm{mm}$ diameter biopsy channel was passed through the nasal passages to the carina; the distal end was then passed into a main-stem bronchus. The bronchial mucosa was topically anesthetized with $2 \%$ lidocaine hydrochloride solution. The tip of the endoscope was inserted in the second branch of the principal right bronchus located ventrally in the right caudal lung lobe (bronchus 1.2). Air from the lavage area was extracted through the bronchoscope biopsy channel by syringe. One hundred milliliters of sterile isotonic saline solution $\left(0.9 \% \mathrm{NaCl}, \mathrm{pH} 6.4,37^{\circ} \mathrm{C}\right)$ were infused under pressure through the bronchoscope biopsy channel into the lung and were immediately aspirated manually. The procedure was repeated 3 times at the same location, giving a total volume of 300 $\mathrm{ml}$.

TW was performed using the same endoscope immediately after BAL according to the method of a previous report [15]. The inside and the outside of the endoscope were washed three times in sterile isotonic saline solution, after which the endoscope was reinserted via the nose to the lowest trachea. TA was collected, followed by injection of $30 \mathrm{ml}$ of sterile saline.

For cytological examination, BALF was mixed with an equal volume of cytospin collection fluid (Thermo Shandon, PA, USA) immediately after collection. Smears were prepared using Cytospin (Thermo Shandon, PA, USA), and were stained by Diff Quick (Baxter, USA). Two hundred cells per specimen were observed using an optical microscope and were classified morphologically. TA was examined using the

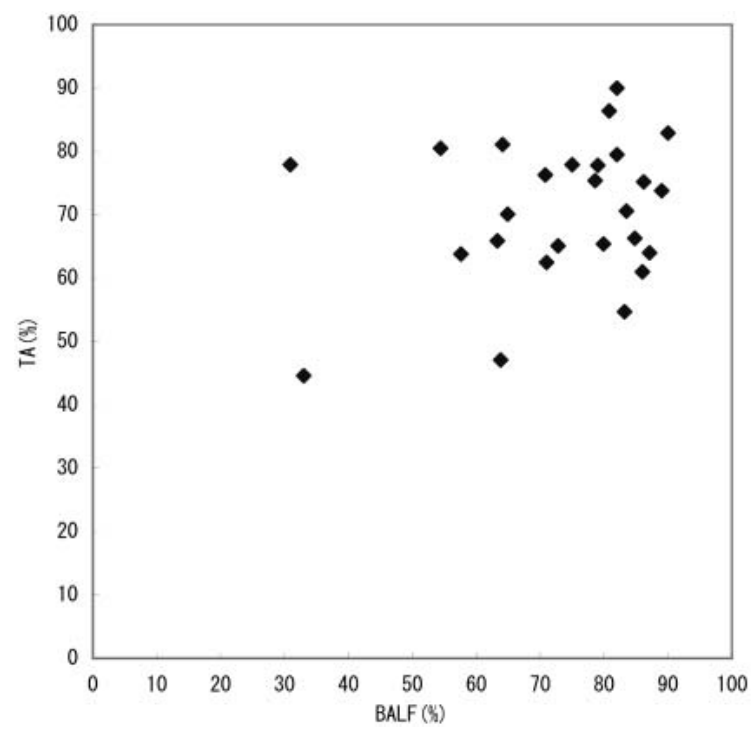

Fig. 1. Comparison of rate $(\%)$ of neutrophils in bronchoalveolar lavage fluid (BALF) and tracheal aspirates (TA) taken from 26 horses at the same time.

same procedure.

For bacteriological examination, a $10 \mathrm{ml}$ aliquot of BALF was centrifuged for $10 \mathrm{~min}$ at 2,000 $\times \mathrm{g}$. After centrifugation, the supernatant was removed and $1 \mathrm{ml}$ of Cary and Blair transport medium without agar was added to the obtained pellets. The samples were stored for bacterial isolation under anaerobic conditions using AneroPack (Mitsubishi Gas Chemical, Tokyo) at $4^{\circ} \mathrm{C}$ until culture. Columbia agar (BD, USA) supplemented with 5\% heparinized horse blood and MacConkey agar (Nissui Seiyaku, Tokyo) were used for bacterial culture. Columbia blood agar plates were prepared in duplicate. One of these and MacConkey agar were incubated aerobically, and the other was incubated anaerobically at $37^{\circ} \mathrm{C}$ for $48 \mathrm{hr}$. Identification of bacterial isolates was carried out using API identification kits (bioMerieux Japan, Tokyo). 
Table 2. Bacterial isolation from bronchoalveolar lavage fluid obtained from racehorses with pneumonia

\begin{tabular}{|c|c|c|c|c|c|c|}
\hline \multirow[t]{2}{*}{ Bacteria } & \multirow{2}{*}{$\begin{array}{l}\text { Isolation } \\
\text { rate }(\%)\end{array}$} & \multicolumn{5}{|c|}{ Number of bacteria $(\mathrm{CFU} / \mathrm{ml})$} \\
\hline & & $<10^{2}$ & $10^{2}-10^{3}$ & $10^{3}-10^{4}$ & $10^{4}-10^{5}$ & $\geq 10^{5}$ \\
\hline Streptococcus zooepidemicus & 39.5 & 5 & 8 & 1 & 1 & 2 \\
\hline Staphylococcus aureus & 2.3 & & & & 1 & \\
\hline Staphylococcus intermedius & 2.3 & & 1 & & & \\
\hline Corynebacterium spp. & 4.7 & & 2 & & & \\
\hline Bacillus spp. & 4.7 & 1 & 1 & & & \\
\hline Nocardia/Streptomyces & 4.7 & 1 & 1 & & & \\
\hline Escherichia coli & 11.3 & 1 & 2 & 2 & & 1 \\
\hline Klebsiella pneumoniae & 4.7 & & & & 1 & 1 \\
\hline Enterobacter cloacae & 2.3 & 1 & & & & \\
\hline Pasteurella pneumotropica & 1.6 & 3 & 3 & 1 & & \\
\hline Pseudomonas aeruginosa & 2.3 & & & 1 & & \\
\hline Stenotrophomonas maltophilia & 2.3 & & 1 & & & \\
\hline Peptostreptococcus spp. & 4.7 & & & & 1 & 1 \\
\hline Gemella morbillorum & 2.3 & 1 & & & & \\
\hline Clostridium perfringens & 2.3 & & 1 & & & \\
\hline Bacteroides uniformis & 2.3 & & & & 1 & \\
\hline Prevotella intermedia & 2.3 & & & & 1 & \\
\hline Prevotella oralis & 2.3 & & & 1 & & \\
\hline Prevotella spp. & 4.7 & & & & 2 & \\
\hline Gram negative obligative anaerobic bacteria & 7.0 & 1 & & 1 & & 1 \\
\hline
\end{tabular}

Bacteria were isolated from 33 of 43 samples of bronchoalveolar lavage fluid, and 1-3 species of bacteria found in large numbers in each sample were identified and counted. Figures in the Table indicate the number of samples from which the bacteria were isolated.

The antimicrobial susceptibility test was performed using the Showa Disc diffusion method according to the manufacturer's provided instructions (Showa Yakuhin Kako, Tokyo). Mueller Hington II agar (BD, USA) was used for this test. The antimicrobial susceptibility of each bacterium tested was judged according to the instruction manual as follows: -, resistant; +, moderately resistant; ++, moderately sensitive; +++, sensitive.

BALF was obtained from 44 horses at the early stage of pneumonia (0-4 days after the onset of treatment). TA was also collected from 26 of the 44 cases using the same bronchoscope immediately after the collection of BAL. Cells in the specimens were classified into neutrophils, macrophages, lymphocytes and epithelial cells. The percentage of neutrophils exceeded $30 \%$ in all samples from BALF and TA, averaging $73.1 \%$ and $70.6 \%$ respectively (Table 1 ). The percentages of neutrophils in both TA and BALF collected from the same horses at the same time were mostly the same and were very high indicating inflammation. A few sample of BALF showed percentages of neutrophils different from TA (Fig. 1).

When BAL is to be performed, it is necessary for the diameter of the bronchi in front of the lesion of pneumonia to match the diameter of the bronchoscope. In this study, a few samples of BALF were collected from areas other than those with pneumonia lesions because of this. It is one BAL's disadvantages as a diagnostic procedure. TW does not suffer from this disadvantage. TA findings appear to give information on the trachea as well as the whole lungs, whereas those of BALF are much more localized.

Bacterial isolation was performed for 43 of 44 BALF samples. More than 20 species of bacteria including obligative anaerobius were isolated from 33 samples (Table 2). S. zooepidemicus predominated $(39.5 \%)$, followed by Escherichia coli (11.3\%), and other bacteria were isolated at rates from 1.6 to $4.7 \%$. BALF samples were then continuously collected from 15 horses to observe the change of the bacterial species and their drug susceptibility. Staphylococcus aureus, Pasteurella pneumotropica, S. zooepidemicus, E. coli and Klebsiella pneumoniae subsp. pneumoniae were isolated predominantly from BALF obtained in the early stages of the clinical course (0-4 days after onset of treatment), and Clostridium spp., Bacteroides spp., Prevotella spp., Pseudomonas aeruginosa, Chryseomonas meningosept, Stenotrophomonas maltophilia, Morganella morganii, Pepotostreptococcus spp. and other obligative 
Table 3. Comparison of species of bacteria, and their drug susceptibility, isolated from bronchoalveolar lavage fluid obtained from 15 racehorses with pneumonia in both the early and later stages of the clinical course

\begin{tabular}{|c|c|c|c|c|c|c|c|c|c|}
\hline \multirow[t]{3}{*}{ Bacteria } & \multicolumn{3}{|c|}{ Drug susceptibility } & \multicolumn{6}{|c|}{ Number of bacteria (CFU/ml) } \\
\hline & \multirow{2}{*}{ Cephalotin } & \multirow{2}{*}{ Gentamicin } & \multirow{2}{*}{ Minocycline } & \multicolumn{3}{|c|}{ in early stage } & \multicolumn{3}{|c|}{ in later stage } \\
\hline & & & & $<10^{3}$ & $10^{3}-10^{5}$ & $\geq 10^{5}$ & $<10^{3}$ & $10^{3}-10^{5}$ & $\geq 10^{5}$ \\
\hline Staphylococcus aureus & +++ & + & +++ & & 1 & & & & \\
\hline Pasteurella pneumotropica & +++ & $+\sim+++$ & +++ & 5 & & & & & \\
\hline Streptococcus zooepidemicus & +++ & $-\sim++$ & $++\sim+++$ & 2 & 1 & 2 & & 1 & \\
\hline Escherichia coli & $+\sim++$ & $++\sim+++$ & $++\sim+++$ & 1 & 2 & 1 & 3 & & \\
\hline Klebsiella pneumoniae & ++ & $++\sim+++$ & ++ & & 1 & 1 & 1 & 1 & \\
\hline Clostridium spp. & +++ & - & $-\sim+++$ & 1 & & & & & 1 \\
\hline Bacteroides spp. & + & - & +++ & & 1 & & 1 & & 1 \\
\hline Prevotella spp. & + & - & ++ & & 1 & & & 2 & 1 \\
\hline $\begin{array}{l}\text { Gram negative obligative } \\
\text { anaerobic rods }\end{array}$ & $\sim \sim+++$ & $-\sim+++$ & $++\sim+++$ & 1 & & & 2 & & 3 \\
\hline Pseudomonas areuginosa & - & $+\sim+++$ & - & & & & 2 & 1 & \\
\hline Chryseomonas meningosept & - & - & ++ & & & & & 1 & \\
\hline Stenotrophomonas maltophilia & - & - & +++ & & & & 2 & & \\
\hline Morganella morganii & - & - & - & & & & & & 1 \\
\hline Peptostreptococcus spp. & +++ & - & +++ & & & & & & 1 \\
\hline $\begin{array}{l}\text { Gram positive obligative } \\
\text { anaerobic cocci }\end{array}$ & +++ & $+\sim++$ & $++\sim+++$ & & & & & & 1 \\
\hline
\end{tabular}

Bronchoalveolar lavage fluid was obtained at 0-4 days (early stage) and at 8-21 days (later stage) after the onset of treatment. Figures in the Table indicate the number of samples from which the bacteria were isolated. Drug susceptibility is shown by:-, resistant; + , moderately resistant; ++, moderately sensitive; +++, sensitive.

anaerobic bacteria were isolated predominantly from BALF obtained in the later stages of the clinical course (8-21 days after onset of treatment) (Table 3). The former bacterial group tended to be more sensitive to cephalotine and gentamicin than the later bacterial group.

S. zooepidemicus has been described as the bacterium isolated most frequently in respiratory diseases of horses $[1,4,12,15]$. In this study, S. zooepidemicus was also isolated at the highest percentage $(39.5 \%)$ from BALF taken from racehorses with pneumonia associated with transport. A study of British racehorses indicated that $S$. zooepidemicus and Streptococcus pneumoniae play an important etiological role in the pathogenesis of IAD in young horses [16]. However, $S$. pneumoniae has not been isolated from BALF or TA in Japan [15]. This may mean that S. pneumoniae is distributed in the horse population in an area including the UK, but not including Japan.

The isolation rates of $S$. zooepidemicus in BALF $(39.5 \%)$ in this study was low in comparison with TA $(83.3 \%)$ taken from young horses with IAD after longdistance transportation without treatment with pharmaceuticals as described in a previous study [15]. Furthermore, the isolation rate of gram-negative obligative anaerobic bacteria tended to increase more in the later stages than in the early stages of the clinical course in this study. This may reflect the effects of antibiotics, since BALs were performed alongside or after the treatment in this study. At the racehorse hospital of the Japan Racing Association, where this study was performed, cephalotin and gentamicin are used as the first choice of therapy for bacterial pneumonia. The results in Table 3 provide evidence of the effect of antimicrobial treatment.

\section{References}

1. Anzai, T., Timoney, J.F., Kuwamoto, Y., Wada, R., Oikawa, M., and Higuchi, T. 2002. Polymerase chain reaction-restriction fragment length polymorphism analysis of the $\mathrm{SzP}$ gene of Streptococcus zooepidemicus isolated from the respiratory tract of horses. Am.J. Vet. Res. 63: 12981301.

2. Beech, J. 1975. Cytology of tracheobronchial aspirates in horses. Vet. Pathol. 12: 157-164.

3. Chapman, P.S., Green, C., Main, J.P.M., Taylor, P.M., Cunningham, F. M., Cook, A.J.C., and Marr, C.M. 2000. Retrospective study of the relationships 
between age, inflammation and the isolation of bacteria from the lower respiratory tract of thoroughbred horses. Vet. Rec. 146: 91-95.

4. Hayakawa, Y., Komae, H., Ide, H., Nakagawa, H., Yoshida, Y., Kamada, M., Kataoka, Y., and Nakazawa, N. 1993. An occurrence of equine transport pneumonia caused by mixed infection with Pasteurella caballi, Streptococcus suis and Streptococcus zooepidemicus. J. Vet. Med. Sci. 55: 455456.

5. Hobo, S., Oikawa, M., Kuwano, A., Yoshida, K., and Yoshihara, T. 1997. Effect of transportation on the composition of bronchoalveolar lavage fluid obtained from horses. Am. J. Vet. Res. 58: 531534.

6. Hoffman, A.M., Viel, L., Muckle, C.A., and Tesarowski, D.B. 1991. Evaluation of a guarded bronchoscopic method for microbial sampling of the lower airways in foals. Can. J. Vet. Res. 55: 325331.

7. Hoffman, A.M., Viel, L., Staempfli, H.R., Muckle, C.A., and Yanger, J.A. 1993. Sensitivity and specificity of bronchoalveolar lavage and protected catheter brush methods for isolating bacteria from foals with experimentally induced pneumonia caused by Klebsiella pneumoniae. Am. J. Vet. Res. 54: 1803-1807.

8. Hughes, K.J., Malikides, N., Hodgson, D.R., and Hodgson, J.L. 2003. Comparison of tracheal aspirates and bronchoalveolar lavage in racehorses 1. Evaluation of cytological stain and the percentage of mast cells and eosinophils. Aus. Vet.J. 81: 681-684.

9. Ito, S., Hobo, S., Eto, D., and Sato, H. 2001. Bronchoalveolar lavage for the diagnosis and treatment of pneumonia associated with transport in thoroughbred racehorses. J. Vet. Med. Sci. 63: 1263-1269.

10. Malikides, N., Hughes, K.J., Hodgson, D.R., and Hodgson, J.L. 2003. Comparison of tracheal aspirates and bronchoalveolar lavage in racehorses 2. Evaluation of the diagnostic significance of neutrophil percentage. Aus. Vet. J. 81: 685-687.

11. Morris, D.D. 1984. Equine tracheobronchial aspirates: Correlation of cytologic and microbiologic findings. J. Am. Vet. Med. Assoc. 184: 340-341.

12. Oikawa, M., Takagi, S., Anzai, T., Yoshikawa, H., and Yoshikawa, T. 1995. Pathology of equine respiratory disease occurring in association with transport. J. Comp. Pathol. 113: 29-43.

13. Robinson, N.E., et al. 2003. Inflammatory airway disease: defining the syndrome. Equine Vet. Educ. 15: $61-63$.

14. Sweeney, C.R., Beech, J., and Roby, K.A.W. 1985. Bacterial isolates from tracheobronchial aspirates of healthy horses. Am. J. Vet. Res. 46: 2562-2565.

15. Takizawa, Y., Hobo, S., Yamauchi, J., Yamane, T., Kuwamoto, Y., Wada, R., and Anzai, T. 2005. Cytological and bacteriological observation of tracheobronchial aspirates from young thoroughbreds transported by vehicle over long distance. J. Equine Sci. 16: 117-121.

16. Wood, J.L.N., Newton, J.R., Chanter N., and Mumford, J.A. 2005. Association between respiratory disease and bacterial and viral infections in British racehorses. J. Clin. Microbiol. 43: 120-126. 